\title{
POSITIVITY PROPERTIES AND STABILITY OF SOLITARY-WAVE SOLUTIONS OF MODEL EQUATIONS FOR LONG WAVES
}

\author{
JOHN P. Albert \\ UNIVERSITY OF OKLAHOMA \\ NORMAN, OK 73019-0315
}

\begin{abstract}
Sufficient conditions are given for stability of solitary-wave solutions of model equations for one-dimensional long nonlinear waves. These conditions differ from others which have appeared previously in that they are phrased in terms of positivity properties of the Fourier transforms of the solitary waves. Their use leads to simplified proofs of existing stability results for the Korteweg-de Vries, BenjaminOno, and Intermediate Long Wave equations; and to new stability results for certain solitary-wave solutions of partial differential equations of Korteweg-de Vries type.
\end{abstract}

\section{Introduction.}

This article is a contribution to the theory of stability of travelling-wave solutions of model equations for one-dimensional long nonlinear waves. Considered herein are equations of the form

$$
u_{t}+u^{p} u_{x}-(M u)_{x}=0,
$$

where $p>0$ is an integer and $M$ is a differential or pseudo-differential operator with positive symbol. (Much of the theory presented below can be extended without difficulty to equations with more general nonlinearities.) The travelling-wave solutions $u(x, t)=\varphi(x-C t)$ of equation (1.1) are usually called solitary waves, in the expectation that the graph of the wave profile $\varphi(x)$ will be dominated by a single hump. For example, the Korteweg-de Vries $(K d V)$ equation

$$
u_{t}+u u_{x}+u_{x x x}=0
$$

has the single-humped travelling-wave solutions $u(x, t)=\varphi_{C}(x-C t)$, where

$$
\varphi_{C}(x)=3 C \operatorname{sech}^{2}\left(\frac{1}{2} \sqrt{C} x\right) .
$$

Theorems on the existence of such solutions for general equations of type (1.1) may be found in [6] and [26].

The mathematically exact stability theory for solitary-wave solutions of equations of type (1.1) dates back to a 1972 paper of Benjamin ([5]), who proved that $K d V$ solitary waves are orbitally stable solutions of the pure initial-value problem 
for equation (1.2). According to Benjamin, if $u(x, t)$ is a solution of (1.2) whose initial profile $u(x, 0)=u_{0}(x)$ is sufficiently close (in an appropriate function space) to a solitary wave profile $\varphi_{C}(x)$; then the quantity

$$
\inf _{y \in \mathbb{R}} \sup _{x \in \mathbb{R}}\left|u(x, t)-\varphi_{C}(x+y)\right|
$$

(which measures the difference in sup norm between the profile $u(x, t)$ for fixed $t$ and the "orbit" consisting of all translates of $\varphi_{C}$ ) will remain small for all times $t>0$.

Similar stability theorems have since been proved for solitary-wave solutions of many other nonlinear wave equations (cf. [10], [13], [14], [26]). A set of sufficient conditions for stability, due to [10] and [26], which appears to be satisfied by solitarywave solutions of a broad class of equations of type (1.1), is the following. Let $\mathcal{L}$ be the linear operator defined on a dense subspace of $L^{2}(\mathbb{R})$ by

$$
\mathcal{L} g(x)=M g(x)+\left(C-\varphi^{p}(x)\right) g(x) .
$$

If $\mathcal{L}$ possesses certain positivity properties - specified below in Theorem 3.1 as properties (P1), (P2), and (P3) - then the (orbital) stability or instability of the solitary wave $\varphi$ is determined by the sign of the quantity $I=\int_{-\infty}^{\infty}\left(\mathcal{L}^{-1} \varphi\right)(x)$. $\varphi(x) d x$.

The central result of this paper is Theorem 3.2, which states that the operator $\mathcal{L}$ possesses the above-mentioned positivity properties (P1)-(P3) whenever the Fourier transform of $\varphi^{p}$ belongs to the class $\mathrm{PF}(2)$ defined by Karlin in [17]. (The relevance to stability theory of this condition on $\varphi$ was first recognized in [2].) As shown in Section 4, Theorem 3.2 leads to a significant simplification of the existing proofs of stability of the solitary-wave solutions of the Korteweg-de Vries, Benjamin-Ono, and Intermediate Long-Wave equations. An important advantage of the present approach is that it does not require an explicit computation of the spectrum of $\mathcal{L}$, such as was needed in [3] and [7]. An instance of how this advantage can lead to new stability results is given in Theorem 4.6 below; which, together with Theorem 4.10, demonstrates the stability of certain solitary-wave solutions of $K d V$ type differential equations with higher-order dispersive terms.

The results of Section 4 may be interpreted as evidence that the hypotheses (P1)-(P3) of Theorem 3.1 hold true for solitary-wave solutions of broad classes of equations of type (1.3). A discussion of this question, together with some preliminary results, is contained in Section 5 .

The plan of the paper is as follows. In section 2, after some remarks on the wellposedness properties of equation (1.1), two families of operators are introduced which play a crucial role in the analysis of the succeeding sections. Section 3 is devoted to the statement and proof of Theorem 3.2, which relates positivity properties of solitary waves to the stability theory of [10]. Applications of Theorem 3.2 to specific solitary waves are presented in Section 4. In the final section appear some general results on positivity properties of solitary waves which are relevant to the theory of section 3 . 


\section{Notation and Preliminaries.}

In this paper, the space of complex-valued functions in $L^{2}(\mathbb{R})$ whose derivatives up to order $s$ also lie in $L^{2}(\mathbb{R})$ will be written as $H^{s}$, and the usual norm on this space is denoted by \|\|$_{s}$. In particular, $H^{0}=L^{2}$, and the usual inner product on $L^{2}$ is denoted by $(,)_{0}$. More generally, if $B$ is any Banach space, the norm on $B$ will be denoted by \|\|$_{B}$. Some of the Banach spaces which will be encountered below are $B\left(L^{2} ; L^{2}\right)$, the space of bounded operators on $L^{2}$; and $C(I, B)$, the space of strongly continuous functions from a real interval $I$ to a Banach space $B$.

The equations considered in this paper are of the form

$$
u_{t}+u^{p} u_{x}-(M u)_{x}=0
$$

where $p>0$ is an integer, and $M$ is defined as a Fourier multiplier operator by

$$
(M g)^{\wedge}(k)=\alpha(k) \hat{g}(k)
$$

for all $k \in \mathbb{R}$. (Here and in what follows, circumflexes will be used to denote Fourier transforms: $\hat{g}(k)=\int_{-\infty}^{\infty} e^{i k x} g(x) d x$.) It is assumed that the symbol $\alpha(k)$ of $M$ is a measurable, locally bounded, even function on $\mathbb{R}$, and satisfies

a) $\quad A_{1}|k|^{\mu} \leq \alpha(k) \leq A_{2}|k|^{\mu} \quad$ for $|k| \geq k_{0}$

b) $\quad \alpha(k) \geq b \quad$ for all $k \in \mathbb{R}$

where $k_{0}$ and $b$ are finite real numbers; $\mu \geq 1$; and $A_{1}, A_{2}>0$.

This paper is concerned only with the pure initial-value problem for $(2.1)$, in which a solution $u: \mathbb{R} \times[0, T) \rightarrow \mathbb{R}$ is sought which has specified initial data $u(x, 0)=u_{0}(x)$. Some well-posedness properties of this problem which will find use below are summarized in the following theorem.

Theorem 2.1. (a) Suppose $s>\frac{3}{2}$. Then for each $u_{0} \in H^{s}$ there exists $T>0$ depending only on $\left\|u_{0}\right\|_{s}$ such that (2.1) has a unique solution $u$ in $C\left([0, T] ; H^{s}\right)$ with $u(x, 0)=u_{0}$. Also, for each $\epsilon>0$ there exists $\delta>0$ such that if $\| \tilde{u}_{0}-$ $u_{0} \|_{s}<\delta$ then (2.1) has a solution $\tilde{u}$ in $C\left([0, T] ; H^{s}\right)$ with $\tilde{u}(x, 0)=\tilde{u}_{0}$, and $\| \tilde{u}-$ $u \|_{C\left([0, T] ; H^{s}\right)}<\epsilon$.

(b) Suppose $s>\frac{3}{2}$. If $p<2 \mu$ and $\mu>3$, then for each $u_{0} \in H^{s}$ there is a unique solution $u$ of (2.1) in $C\left([0, \infty) ; H^{s}\right)$ with $u(x, 0)=u_{0}$.

The proofs of Theorems 2.1(a) and (b), which are somewhat lengthy and which make use only of standard techniques, will not be given here. Theorem 2.1(a) is a consequence of the local existence theory put forth in [19]; similar results are to be found in [1]. Theorem 2.1(b) is deduced from 2.1(a) in the usual way by using conservation laws for equation (2.1) to derive a priori bounds on $\|u\|_{s}$.

Let $u(x, t)=\varphi(x-C t)$ be a travelling-wave solution of (2.1). Substituting this form of $u(x, t)$ into (2.1) and integrating once (with zero boundary conditions imposed at infinity), one obtains

$$
(M+C) \varphi=\left(\frac{1}{p+1}\right) \varphi^{p+1} .
$$


Any solution $\varphi$ of (2.3) which is an even function and which lies in the space $H^{\mu / 2}$ will henceforth be termed a solitary wave. It will also be assumed in what follows that the wavespeed $C$ satisfies $C>-b$, where $b$ is the constant appearing in (2.2)b: hence $M+C$ represents a positive operator. An immediate consequence of these assumptions, which may be established by a simple "bootstrap" argument, is that a solitary wave $\varphi$ must lie in $H^{s}$ for all $s \in \mathbb{R}$. Hence $\varphi$ is infinitely differentiable, with all derivatives in $L^{2}$.

In studying the stability of the solitary wave $\varphi$, it has been found useful to consider the linear operator $\mathcal{L}: L^{2} \rightarrow L^{2}$ defined by

$$
\mathcal{L} u=(M+C) u-\varphi^{p} \cdot u .
$$

Proposition 2.1. The operator $\mathcal{L}$ is a closed, unbounded, self-adjoint operator on $L^{2}$ whose spectrum consists of the continuous part $[C, \infty)$ together with a finite number of discrete eigenvalues (with finite-dimensional eigenspaces) in the interval $(-\infty, C]$. In particular, $\mathcal{L}$ has the eigenvalue 0 , with eigenfunction $d \varphi / d x$.

Proof. See [3], Proposition 1.

Much of the remainder of this paper is concerned with a finer study of the spectral properties of $\mathcal{L}$. For this purpose, it has been found convenient to consider two families of linear operators which are closely related to $\mathcal{L}$, but have the added advantage of being compact and bounded.

For each $\theta \geq 0$, define the operator $S_{\theta}$ on $L^{2}(\mathbb{R})$ by

$$
S_{\theta} g(x)=\left(\frac{1}{w_{\theta}(x)}\right) \int_{-\infty}^{\infty} K(x-y) g(y) d y,
$$

where $K(x)=\left(\varphi^{p}\right)^{\wedge}(x)$ and $w_{\theta}(x)=\alpha(x)+\theta+C$. Since $w_{\theta}(x)>0$ for all $x \in \mathbb{R}$, it follows that the space $X$ defined by

$$
X=\left\{g \in L^{2}:\|g\|_{X, \theta}=\left(\int_{-\infty}^{\infty}|g(x)|^{2} w_{\theta}(x) d x\right)^{\frac{1}{2}}<\infty\right\}
$$

is a Hilbert space with norm $\|g\|_{X, \theta}$ and corresponding inner product

$$
\langle g, h\rangle_{X, \theta}=\int_{-\infty}^{\infty} g(x) \overline{h(x)} w_{\theta}(x) d x .
$$

(In fact, the underlying space $X$ is just $H^{\mu / 2}$, and each of the norms $\|g\|_{X, \theta}$ is equivalent to the standard norm $\|g\|_{\mu / 2}$.)

Proposition 2.2. a) If $g \in L^{2}$ is an eigenfunction of $S_{\theta}$ for a non-zero eigenvalue, then $g \in X$.

b) The restriction of $S_{\theta}$ to $X$ is a compact, self-adjoint operator on $X$ with respect to the norm \|\|$_{X, \theta}$.

Proof. See [2], Lemmas 6 and 7, for the proof in the case $\theta=0$. The proof for the case $\theta>0$ is identical.

The following two corollaries are immediate consequences of Proposition 2.2 and the spectral theorem for self-adjoint compact operators on a Hilbert space. 
Corollary 2.3. Suppose $\theta \geq 0$. Then 1 is an eigenvalue of $S_{\theta}$ (as an operator on $X$ ) if and only if $-\theta$ is an eigenvalue of $\mathcal{L}$ (as an operator on $L_{2}$ ). Furthermore both eigenvalues have the same multiplicity.

Corollary 2.4. For every $\theta \geq 0, S_{\theta}$ has a family of eigenvectors $\left\{\psi_{i}(\theta)\right\}_{i=1}^{\infty}$ forming an orthonormal basis of $X$ with respect to the norm \|\|$_{X, \theta}$. The eigenvectors correspond to real eigenvalues $\left\{\lambda_{i}(\theta)\right\}_{i=1}^{\infty}$ whose only possible accumulation point is zero.

The eigenvalues $\lambda_{i}(\theta)$ will henceforth be numbered in order of decreasing absolute value, so that $\left|\lambda_{1}\right| \geq\left|\lambda_{2}\right| \geq \cdots \geq 0$.

The second family of operators which will find use below is the family $\left\{T_{\theta}\right\}_{\theta \geq 0}$ defined by

$$
T_{\theta} g=(M+C+\theta)^{-1}\left(\varphi^{p} \cdot g\right) .
$$

When working with $T_{\theta}$ it will always be assumed that the solitary wave profile $\varphi$ is everywhere positive. The operator $T_{\theta}$ is viewed as acting on the Hilbert space

$$
Y=\left\{g: g \text { is measurable on } \mathbb{R} \text { and }\|g\|_{Y}=\left(\int_{-\infty}^{\infty}|g|^{2} \varphi^{p} d x\right)^{\frac{1}{2}}<\infty\right\},
$$

which is furnished with the inner product $\langle g, h\rangle_{Y}=\int_{-\infty}^{\infty} g(x) \overline{h(x)} \varphi^{p}(x) d x$. The action of $T_{\theta}$ on $Y$ is described in the next two Propositions.

Proposition 2.5. a) If $g \in Y$ is an eigenfunction of $T_{\theta}$ for a non-zero eigenvalue, then $g \in L_{2}$.

b) The operator $T_{\theta}$ is a self-adjoint compact operator on $Y$.

Proof. a) If $T_{\theta} g=\lambda g$ with $\lambda \neq 0$, then $\hat{g}=\frac{1}{\lambda} \cdot \frac{1}{w_{\theta}} \cdot\left(\varphi^{p} g\right)^{\wedge}$. Since $g \in Y$, then $\varphi^{p} g \in L_{2}$, and it follows that $\hat{g} \in L_{2}$. Hence $g \in L_{2}$.

b) The action of $T_{\theta}$ on $Y$ is given by $T_{\theta} g(x)=\int_{-\infty}^{\infty} G_{\theta}(x-y) g(y) d \nu(y)$, where $d \nu(y)$ is the positive measure on $\mathbb{R}$ defined by $d \nu(y)=\varphi^{p}(y) d y$, and $G_{\theta}(x)=$ $\left(w_{\theta}^{-1}\right)^{\wedge}(x)$. From $(2.2)$ it follows that $\left(w_{\theta}\right)^{-1} \in L_{2} ;$ so $G_{\theta} \in L_{2}$ and hence $G_{\theta}(x-$ $y) \in L_{2}(d \nu(x) \times d \nu(y))$. Therefore $T_{\theta}$ is a Hilbert-Schmidt operator on $Y$, so $T_{\theta}$ is bounded and compact on $Y$. Moreover, since $\alpha(k)$ is even, so are $w_{\theta}$ and $G_{\theta}$; and it follows that $T_{\theta}$ is self-adjoint on $Y$.

Proposition 2.6. Suppose $\operatorname{ker}\left(T_{\theta}\right)=0$. Let $\left\{\zeta_{i}\right\}_{i=0}^{\infty}$ be a complete orthonormal set of eigenfunctions of $T_{\theta}$ in $Y$, with $T_{\theta} \zeta_{i}=\lambda_{i} \zeta_{i}$ for $i \geq 0$. Then $\left\{\sqrt{\lambda_{i}} \hat{\zeta}_{i}\right\}_{i=0}^{\infty}$ is a complete orthonormal set of eigenfunctions for $S_{\theta}$ in $X$, with $S_{\theta} \hat{\zeta}_{i}=\lambda_{i} \hat{\zeta}_{i}$.

Proof. For $i \geq 0, \zeta_{i}$ (and hence also $\hat{\zeta}_{i}$ ) is in $L_{2}$ by Proposition 2.5. From the definitions of $S_{\theta}$ and $T_{\theta}$ it follows that $S_{\theta}\left(\hat{\zeta}_{i}\right)=\left(T_{\theta} \zeta_{i}\right)^{\wedge}=\lambda_{i} \hat{\zeta}_{i}$. Hence, by Proposition 2.2, $\hat{\zeta}_{i} \in X$. The orthogonality of the $\hat{\zeta}_{i}$ in $X$ follows from the self-adjointness of $S_{\theta}$ on $X$. To see that $\left\{\sqrt{\lambda_{i}} \hat{\zeta}_{i}\right\}_{i=0}^{\infty}$ forms an orthonormal set; note that since $\operatorname{ker}\left(T_{\theta}\right)=0$, one has $\lambda_{i} \neq 0$ for all $i$. Then a simple computation shows that 
for all $g \in X,\left\langle g, \hat{\zeta}_{i}\right\rangle_{X, \theta}=\lambda_{i}^{-1}\left\langle g, S_{\theta} \hat{\zeta}_{i}\right\rangle_{X, \theta}=\lambda_{i}^{-1}\left\langle\hat{g}, \zeta_{i}\right\rangle_{Y}$. Setting $g=\lambda_{i} \hat{\zeta}_{i}$ gives $\left\langle\lambda_{i} \hat{\zeta}_{i}, \hat{\zeta}_{i}\right\rangle_{X, \theta}=\left\langle\zeta_{i}, \zeta_{i}\right\rangle_{Y}=1$, showing that $\left\|\sqrt{\lambda_{i}} \hat{\zeta}_{i}\right\|_{X, \theta}=1$.

It remains to show that $\left\{\sqrt{\lambda_{i}} \hat{\zeta}_{i}\right\}_{i=0}^{\infty}$ is complete in $X$. Suppose $g \in X$ and $\left\langle g, \hat{\zeta}_{i}\right\rangle_{X, \theta}=0$ for all $i \geq 0$. Then $\left\langle\hat{g}, \zeta_{i}\right\rangle_{Y}=\lambda_{i}\left\langle g, \hat{\zeta}_{i}\right\rangle_{X, \theta}=0$ for all $i \geq 0$; and from the completeness of $\left\{\zeta_{i}\right\}_{i=0}^{\infty}$ in $Y$ it follows that $\hat{g}=0$, and hence that $g=0$.

This section concludes with a brief account of certain facts concerning Gegenbauer polynomials which will find application in Section 4.

Let $\rho>-\frac{1}{2}$ be a given real number. The Gegenbauer polynomials $\left\{C_{m}^{\rho}\right\}_{m=0}^{\infty}$ are defined by

$$
C_{m}^{\rho}(\xi)=\sum_{s=0}^{[m / 2]}(-1)^{s} \frac{\Gamma(m+\rho-s)}{s !(m-2 s) ! \Gamma(\rho)}(2 \xi)^{m-2 s} .
$$

(The expression for the coefficients of $C_{m}^{\rho}$ is not defined if $\rho=0$, but this case will not arise below.) Let $L_{2, \rho}=L_{2, \rho}([-1,1])$ be the space of all measurable functions $h$ on $[-1,1]$ such that $\|h\|_{2, \rho}=\left(\int_{-1}^{1}|h(\xi)|^{2}\left(1-\xi^{2}\right)^{\rho-\frac{1}{2}} d \xi\right)^{\frac{1}{2}}<\infty$. Then $L_{2, \rho}$ is a Hilbert space with the inner product $\langle g, h\rangle_{2, \rho}=\int_{-1}^{1} g(\xi) \overline{h(\xi)}\left(1-\xi^{2}\right)^{\rho-\frac{1}{2}} d \xi$; and $\left\{C_{m}^{\rho}\right\}_{m=0}^{\infty}$ forms a complete orthogonal set in $L_{2, \rho}$; with normalizing constants given by

$$
\left\|C_{m}^{\rho}\right\|_{2, \rho}=\left\{\frac{\pi 2^{1-2 \rho} \Gamma(m+2 \rho)}{\Gamma(\rho)^{2}(m+\rho) m !}\right\}^{\frac{1}{2}}
$$

(Here $\Gamma$ denotes Euler's Gamma function.) If $\left\{P_{m}\right\}_{m=0}^{\infty}$ is any other set of orthogonal polynomials in $L_{2, \rho}$ such that $\operatorname{deg}\left(P_{m}\right)=m$ for all $m \geq 0$, then each $P_{m}$ must be a constant multiple of $C_{m}^{\rho}$. Finally, for all $\rho, \sigma>-\frac{1}{2}$ one has the identity

$$
\int_{-1}^{1} C_{m}^{\rho}(\xi)\left(1-\xi^{2}\right)^{\sigma-\frac{1}{2}} d \xi= \begin{cases}{\left[\frac{\Gamma\left(\sigma+\frac{1}{2}\right)}{\sqrt{\pi} \Gamma(\rho) \Gamma(\rho-\sigma)}\right]\left[\frac{\Gamma\left(\frac{m}{2}+\rho-\sigma\right) \Gamma\left(\frac{m}{2}+\rho\right)}{\Gamma\left(\frac{m}{2}+1\right) \Gamma\left(\frac{m}{2}+\sigma+1\right)}\right]} & (\text { for } m \text { even }) \\ 0 & \text { (for } m \text { odd })\end{cases}
$$

For proofs of the above facts concerning Gegenbauer polynomials, the reader is referred to [15] and [23].

\section{Stability and Positivity Properties of Solitary waves.}

If $\varphi$ is a given solitary-wave solution of (2.1); define the set $\mathcal{O}_{\varphi} \subseteq X$ by $\mathcal{O}_{\varphi}=$ $\{g: g(\cdot)=\varphi(\cdot+r)$ for some $r \in \mathbb{R}\}$ and for any $\eta>0$ define the set $U_{\eta} \subseteq X$ by $U_{\eta}=$ $\left\{g: \inf _{h \in \mathcal{O}_{\varphi}}\|g-h\|_{X, 0}<\eta\right\}$.

With this terminology, $\varphi$ is defined to be (orbitally) stable if

(i) there exists an $s_{0}>\frac{3}{2}$ and a neighborhood $U$ of $\varphi$ in $H^{s_{0}}$ such that for all $u_{0} \in U$, there is a unique solution $u$ of $(2.1)$ in $C\left([0, \infty) ; H^{s_{0}}\right)$ with $u(x, 0)=u_{0} ; \quad$ and

(ii) for every $\epsilon>0$, there exists a $\delta>0$ such that for all $u_{0} \in U_{\delta} \cap U$, the solution $u$ of $(2.1)$ with $u(x, 0)=u_{0}$ satisfies $u(\cdot, t) \in U_{\epsilon}$ for all $t>0$. 
The following Theorem is proved in [10] (see also [2], [26]):

Theorem 3.1. Let $\varphi$ be a solitary-wave solution of (2.1), and suppose that part (i) of the definition of stability holds for $\varphi$. Suppose also that the operator $\mathcal{L}$ defined in the preceding section has the following properties:

(P1) $\mathcal{L}$ has a simple, negative eigenvalue $\kappa$,

$(\mathrm{P} 2) \mathcal{L}$ has no negative eigenvalue other than $\kappa$, and

(P3) the eigenvalue 0 of $\mathcal{L}$ is simple.

Choose $\psi \in L^{2}$ such that $\mathcal{L} \psi=\varphi$, and define $I=(\psi, \varphi)_{0}$. If $I<0$, then $\varphi$ is stable.

Remarks. (i) By assumption (P3), the nullspace $N$ of $\mathcal{L}$ is the one-dimensional subspace of $L^{2}$ spanned by $d \varphi / d x$. Since $(\varphi, d \varphi / d x)_{0}=0$, it follows that $\varphi \perp N$ in $L^{2}$. Therefore, by the self-adjointness of $\mathcal{L}$, the set $\mathcal{L}^{-1}(\varphi)$ is nonempty, and the value of $I$ does not depend on the choice of $\psi$ in $\mathcal{L}^{-1}(\varphi)$.

(ii) Recently, Souganides and Strauss have shown ([24], see also [10]) that under assumptions (P1)-(P3), and certain mild restrictions on the symbol $\alpha(k)$ of $M$, the positivity of $I$ implies the instability of $\varphi$.

The verification of $(\mathrm{P} 1)-(\mathrm{P} 3)$ directly for the operator $\mathcal{L}$ is in general not an easy task (see, for example, [3] and [7]). An alternate approach which has proved fruitful is to deduce (P1)-(P3) from a spectral analysis of the operators $S_{\theta}$ defined in the previous section. It transpires that $(\mathrm{P} 1)-(\mathrm{P} 3)$ are consequences of a certain positivity condition on the function $K$ appearing in the kernels of the $S_{\theta}$. Following the terminology of [17], one says that a function $g: \mathbb{R} \rightarrow \mathbb{R}$ is in the class $P F(2)$ if

(i) $g(x)>0$ for $x \in \mathbb{R}$

(ii) $g\left(x_{1}-y_{1}\right) g\left(x_{2}-y_{2}\right)-g\left(x_{1}-y_{2}\right) g\left(x_{2}-y_{1}\right) \geq 0$ for $x_{1}<x_{2}$ and $y_{1}<y_{2}$; and

(iii) strict inequality holds in (ii) whenever the intervals $\left(x_{1}, x_{2}\right)$ and $\left(y_{1}, y_{2}\right)$ intersect.

Theorem 3.2. Suppose $\hat{\varphi}>0$ on $\mathbb{R}$ and $\left(\varphi^{p}\right)^{\wedge}=K \in P F(2)$. Then (P1), (P2), and (P3) hold for the operator $\mathcal{L}$.

Proof. As was shown in Lemma 10 and the proof of Theorem 4 of [2], the stated assumptions on $\varphi$ and $K$ imply that for all $\theta \geq 0$, the eigenvalues $\lambda_{0}(\theta)$ and $\lambda_{1}(\theta)$ of $S_{\theta}$ are distinct, positive and simple. Moreover, by classical perturbation theory (see [18]), $\lambda_{0}(\theta)$ and $\lambda_{1}(\theta)$ depend differentiably on $\theta$ in $[0, \infty)$; and corresponding eigenfunctions $\psi_{0}=\psi_{0}(\theta) \in X$ and $\psi_{1}=\psi_{1}(\theta) \in X$ may be chosen which also depend differentiably on $\theta$ in $[0, \infty)$ and which satisfy $\left\|\psi_{0}(\theta)\right\|_{X, \theta}=\left\|\psi_{1}(\theta)\right\|_{X, \theta}=1$ for all $\theta \geq 0$.

It is now claimed that

$$
\frac{d}{d \theta}\left(\lambda_{i}(\theta)\right)<0 \text { for } i=0,1 \text { and } \theta \geq 0
$$


This may be proved by the following computation:

$$
\begin{aligned}
\frac{d \lambda_{i}}{d \theta} & =\frac{d}{d \theta}\left(\lambda_{i}\left\|\psi_{i}\right\|_{X, \theta}\right) \\
& =\frac{d}{d \theta}\left\{\lambda_{i} \int_{-\infty}^{\infty}\left(\psi_{i}(x)\right)^{2} w_{\theta}(x) d x\right\} \\
& =\frac{d}{d \theta}\left\{\int_{-\infty}^{\infty}\left(S_{\theta} \psi_{i}(x)\right) \psi_{i}(x) w_{\theta}(x) d x\right\} \\
& =\frac{d}{d \theta}\left\{\int_{-\infty}^{\infty}\left(\int_{-\infty}^{\infty} K(x-y) \psi_{i}(y) d y\right) \psi_{i}(x) d x\right\} \\
& =\int_{-\infty}^{\infty} \int_{-\infty}^{\infty} K(x-y)\left\{\frac{d \psi_{i}}{d \theta}(y) \psi_{i}(x)+\psi_{i}(y) \frac{d \psi_{i}}{d \theta}(x)\right\} d x d y \\
& =2 \int_{-\infty}^{\infty} \frac{d \psi_{i}}{d \theta}(x)\left(S_{\theta} \psi_{i}(x)\right) w_{\theta}(x) d x \\
& =2 \lambda_{i} \int_{-\infty}^{\infty} \frac{d \psi_{i}}{d \theta}(x) \psi_{i}(x) w_{\theta}(x) d x \\
& =2 \lambda_{i}\left\{\frac{d}{d \theta}\left(\frac{1}{2} \int_{-\infty}^{\infty}\left(\psi_{i}(x)\right)^{2} w_{\theta}(x) d x\right)-\frac{1}{2} \int_{-\infty}^{\infty}\left(\psi_{i}(x)\right)^{2} d x\right\} \\
& =2 \lambda_{i}\left\{0-\frac{1}{2} \int_{-\infty}^{\infty}\left(\psi_{i}(x)\right)^{2} d x\right\} \\
& =-\lambda_{i} \int_{-\infty}^{\infty}\left(\psi_{i}(x)\right)^{2} d x<0 .
\end{aligned}
$$

(Here use has been made of the symmetry of $K$ and of the fact that $\frac{d}{d \theta}\left(w_{\theta}(x)\right)=$ $\frac{d}{d \theta}(\alpha(x)+\theta+C)=1$.)

Next, notice that for all $\theta \geq 0,\left|\lambda_{0}(\theta)\right|=\left(\right.$ spectral radius of $\left.S_{\theta}\right) \leq\left\|S_{\theta}\right\|_{B\left(L^{2} ; L^{2}\right)}=$ $\left(\int_{-\infty}^{\infty} \int_{-\infty}^{\infty}\left\{\frac{K(x-y)}{w_{\theta}(x)}\right\}^{2} d x d y\right)^{\frac{1}{2}}=\|K\|_{L^{2}} \int_{-\infty}^{\infty}\left(\frac{1}{w_{\theta}(x)}\right)^{2} d x$. It follows from the Dominated Convergence Theorem that

$$
\lim _{\theta \rightarrow \infty} \lambda_{0}(\theta)=0
$$

Now from the proof of Theorem 4 of [2], one has

$$
\lambda_{1}(0)=1
$$

Hence $\lambda_{0}(0)>1$, and from (3.1) and (3.2) it then follows that there exists a unique $\theta_{0} \in(0, \infty)$ such that $\lambda_{0}\left(\theta_{0}\right)=1$. One then obtains (P1) by setting $\kappa=-\theta_{0}$ and applying Corollary 2.3. Also, for $i \geq 1$ and $\theta>0$, one has $\lambda_{i}(\theta) \leq \lambda_{1}(\theta)<\lambda_{1}(0)=$ 1 ; showing that 1 is not an eigenvalue of $S_{\theta}$ for any positive value of $\theta$ besides $\theta=\theta_{0}$. Another application of Corollary 2.3 then gives (P2). Finally, (P3) is an immediate consequence of Corollary 2.3 and (3.3). 


\section{Examples.}

This section begins by showing that the theory of the preceding section may be used to recover, via a simple, unified approach, several existing results on the stability of solitary waves (see Theorem 4.4 below). The remainder of the section shows how Theorems 3.1 and 3.2 may be applied to a certain family of partial differential equations of type (2.1) which have special solitary-wave solutions of a simple form.

The well-known Korteweg-de Vries $(K d V)$ equation, given by $(2.1)$ with $p=1$ and $\alpha(k)=k^{2}$, has the family of solitary-wave solutions $\left\{\varphi_{C}^{K d V}\right\}_{C>0}$, where $C$ is the wavespeed and $\varphi_{C}^{K d V}(x)=3 C \operatorname{sech}^{2}\left(\frac{1}{2} \sqrt{C} x\right)$. The Benjamin-Ono $(B O)$ equation has $p=1, \alpha(k)=|k|$, and the family of solitary-wave solutions discovered by Benjamin in [4] and given by $\varphi_{C}^{B O}(x)=4 C /\left(1+C^{2} x^{2}\right)$ (where $\left.C>0\right)$. Interpolating between the $K d V$ and $B O$ equations (in a sense which was made precise in [1]) is a family of equations depending on the positive parameter $H$, and known collectively as the Intermediate Long Wave $(I L W)$ equation. For $I L W$, one has $p=1, \alpha(k)=$ $k \operatorname{coth} k H-\frac{1}{H}$, and, for each $H \in(0, \infty)$, the family of solitary-wave solutions $\left\{\varphi_{C, H}^{I L W}\right\}_{C>0}$ discovered by Joseph ([16]). For a given $H>0$ and $C>0$, if $a \in$ $\left(0, \frac{\pi}{2 H}\right)$ and $b \in(0, \infty)$ are defined by the equations

$$
\begin{gathered}
1-a H \tan a H+a H \tan a H=C H \quad \text { and } \\
\frac{b H}{4}=a H \tan a H,
\end{gathered}
$$

then

$$
\varphi_{C, H}^{I L W}(x)=\frac{b}{\left(\cosh ^{2}(a x)+\left(\frac{b^{2}}{16 a^{2}}\right) \sinh ^{2}(a x)\right)} .
$$

Lemma 4.1. ([10],[25]) Suppose $\left\{\varphi_{C}\right\}_{C>0}$ is a family of solutions of (2.3) such that the correspondence $C \mapsto \varphi_{C}$ determines a differentiable map from $\mathbb{R}^{+}$to $H^{\mu / 2}$. Then the quantity I defined in Theorem 3.1 is given by the formula

$$
I=-\frac{1}{2} \frac{d}{d C}\left(\left\|\varphi_{C}\right\|_{L^{2}}^{2}\right) .
$$

Proof. Substituting $\varphi_{C}$ in $(2.3)$ and differentiating with respect to $C$ yields the equation $\mathcal{L}\left(\frac{-d\left(\varphi_{C}\right)}{d C}\right)=-\varphi_{C}$. Therefore

$$
I=\int_{-\infty}^{\infty}\left(\frac{-d\left(\varphi_{C}\right)}{d C}\right) \cdot \varphi d x=\frac{-1}{2} \frac{d}{d C}\left(\left\|\varphi_{C}\right\|_{L^{2}}^{2}\right) .
$$

Lemma 4.2. For the solitary waves $\varphi_{C}^{K d V}, \varphi_{C}^{B O}$, and $\varphi_{C, H}^{I L W}$; one has $I<0$ for all $C>0$ and $H>0$.

Proof. Since $\left\|\varphi_{C}^{K d V}\right\|_{L^{2}}^{2}=C^{3 / 2}\left\|\varphi_{1}^{K d V}\right\|_{L^{2}}^{2}$ and $\left\|\varphi_{C}^{B O}\right\|_{L^{2}}^{2}=C\left\|\varphi_{1}^{B O}\right\|_{L^{2}}^{2}$, the result for $\varphi_{C}^{K d V}$ and $\varphi_{C}^{B O}$ follows immediately from Lemma 4.1. The result for $\varphi_{C, H}^{I L W}$ may 
also be obtained from Lemma 4.1 by explicitly computing $\left\|\varphi_{C, H}^{I L W}\right\|_{L^{2}}^{2}$; for details the reader is referred to the proof of Theorem 11 of [2].

Lemma 4.3. Suppose $f$ is a positive, twice-differentiable function on $\mathbb{R}$ satisfying $\frac{d^{2}}{d x^{2}}(\log f)<0$ for $x \neq 0$. Then $f \in P F(2)$.

Proof. See Lemma 10 of [2].

Theorem 4.4. ([2], [5], [7], [8]) The solitary waves $\varphi_{C}^{K d V}, \varphi_{C}^{B O}$, and $\varphi_{C, H}^{I L W}$ are stable for all $C>0$ and all $H>0$.

Proof. For these solitary waves, part (i) of the definition of stability given in section 3 is proved in [1]. To prove part (ii), by Theorems 3.1 and 3.2 and Lemma 4.2, it suffices to show that $\left(\varphi_{C}^{K d V}\right)^{\wedge},\left(\varphi_{C}^{B O}\right)^{\wedge}$ and $\left(\varphi_{C, H}^{I L W}\right)^{\wedge}$ are in $P F(2)$.

A table of Fourier transforms yields that

$$
\begin{aligned}
\left(\varphi_{C}^{K d V}\right)^{\wedge}(x) & =\frac{12 \pi x}{\sinh \left(\frac{\pi x}{\sqrt{C}}\right)} \\
\left(\varphi_{C}^{B O}\right)^{\wedge}(x) & =4 \pi e^{-|C x|}, \quad \text { and } \\
\left(\varphi_{C, H}^{I L W}\right)^{\wedge}(x) & =2 \pi \frac{\sinh \left(\frac{\delta x}{2 a}\right)}{\sinh \left(\frac{\pi x}{2 a}\right)}
\end{aligned}
$$

where $\delta \in(-\pi, \pi)$ is such that $\cos \delta=\left(\frac{16 a^{2}-b^{2}}{16 a^{2}+b^{2}}\right)$. Using the formula for $\left(\varphi_{C}^{B O}\right)^{\wedge}$, it is easy to verify directly that $\left(\varphi_{C}^{B O}\right)^{\wedge}$ satisfies the definition of $P F(2)$ given above in Section 3. The fact that $\left(\varphi_{C}^{K d V}\right)^{\wedge}$ and $\left(\varphi_{C, H}^{I L W}\right)^{\wedge}$ are in $P F(2)$ follows easily from Lemma 4.3 .

The stability result for the $K d V$ solitary wave contained in the preceding theorem has been extended to solitary-wave solutions of the generalized $K d V$ equation

$$
u_{t}+u^{p} u_{x}+u_{x x x}=0
$$

In fact, in [10] it is shown that the solitary-wave solutions of this equation are stable if and only if $p<4$ (see also [25]). In the remainder of this section, it will be shown how Theorems 3.1 and 3.2 may be used to generalize this result to treat certain solitary-wave solutions of equations of the form

$$
u_{t}+u^{p} u_{x}-\left(M_{n, p}(u)\right)_{x}=0
$$

where $M_{n, p}$ is a differential operator of order $2 n$. The solitary waves in question are of the form $\varphi(x)=(\operatorname{sech}(x))^{r}$, where $r=\frac{2 n}{p}$. In the several propositions which follow, the symbols $\varphi$ and $r$ will be given these connotations consistently.

The operators $M_{n, p}$ will be defined by means of the following Proposition. 
Proposition 4.5. Let $n$ be a given positive integer and $p$ a given positive real number. Then there exists a unique vector $A=\left(a_{0}, a_{1}, \ldots, a_{n}\right)$ in $\mathbb{R}^{n+1}$ such that

$$
\sum_{i=0}^{n} a_{i}\left(\partial^{2 i} \varphi\right)=\frac{\varphi^{p+1}}{p+1}
$$

Proof. For each natural number $i$, one has $\partial^{2 i} \varphi=\sum_{j=0}^{i} b_{i j} \operatorname{sech}^{r+2 j}(x)$, where the $b_{i j}$ are non-zero real numbers depending only on $r$. Define $B$ to be the $(n+$ $1) \times(n+1)$ matrix $\left\{b_{i j}\right\}_{i, j=0, n}$, where $b_{i j}$ is set equal to zero for $i<j$. Since $\varphi^{p+1}(x)=\operatorname{sech}^{r+2 n}(x)$, equation (4.1) holds if and only if $A B=D$; where $D=$ $\left(0,0, \ldots, 0, \frac{1}{p+1}\right) \in \mathbb{R}^{n+1}$. But $B$ is non-singular, as it is a lower-diagonal matrix with non-zero elements on the diagonal. Hence there is a unique $A$ in $\mathbb{R}^{n+1}$ for which (4.1) holds.

Now, for given $n$ and $p$, define the differential operator $M_{n, p}$ by

$$
M_{n, p}=\sum_{i=1}^{n} a_{i} \partial^{2 i}
$$

where the $a_{i}$ are the constants appearing in Proposition 4.5. Also define $C_{n, p}=a_{0}$. Then by (4.1), one has

$$
\left(M_{n, p}+C_{n, p}\right)(\varphi)=\frac{1}{p+1}(\varphi)^{p+1}
$$

Hence $\varphi$ is a solution of (2.3) with $M=M_{n, p}$ and $C=C_{n, p}$. In order to apply the theorems of section 3 to the solitary wave $\varphi$, one must verify the condition $C>-\inf _{k \in \mathbb{R}} \alpha(k)$; where $\alpha(k)$ is the symbol of $M_{n, p}$. To see this, notice that for any $\nu \in \mathbb{R}^{+}$, the Fourier transform of $\left(\operatorname{sech}^{\nu}(x)\right)$ is given by $\int_{-\infty}^{\infty} e^{i k x}\left(\operatorname{sech}^{\nu}(x)\right) d x=$ $2^{\nu-1}(\Gamma(\nu))^{-1}\left|\Gamma\left(\frac{\nu}{2}+i \frac{k}{2}\right)\right|^{2}$, which is a positive function of $k \in \mathbb{R}([21$, p. 34]). In particular, $\hat{\varphi}(k)$ and $\left(\varphi^{p+1}\right)^{\wedge}(k)$ are everywhere positive; and from (4.2) it follows that

$$
\alpha(k)+C_{n, p}=\frac{1}{p+1} \frac{\left(\varphi^{p+1}\right)^{\wedge}(k)}{\hat{\varphi}(k)}>0
$$

for all $k \in \mathbb{R}$. Since $\alpha(k)$ is an even polynomial, this implies $\left(\inf _{k \in \mathbb{R}} \alpha(k)\right)+C_{n, p}>0$, as desired.

Theorem 4.6. Let $n$ be a positive integer and suppose $0<p<4 n$. Consider the solitary-wave solution $\varphi(x)=(\operatorname{sech}(x))^{r}$ of equation (2.1), where $M=M_{n, p}$ and $r=\frac{2 n}{p}$. If the quantity $I$ defined in Theorem 3.1 satisfies $I<0$, then $\varphi$ is a stable solution of (2.1).

Proof. The validity of part (i) of the definition of stability of $\varphi$ in section 3 was established in [19] for the case $n=1$. If $n \geq 2$, then (2.2) holds with $\mu=2 n \geq 4$, so that part (i) holds true by Theorem 2.1(b). 
Next consider the function $K=\left(\varphi^{p}\right)^{\wedge}=\left(\operatorname{sech}^{2 n}(x)\right)^{\wedge}$. The function

$$
\left(\operatorname{sech}^{2}(x)\right)^{\wedge}=\frac{\pi x}{\sinh \left(\frac{\pi x}{2}\right)}
$$

is "logarithmically concave"; which is to say, it satisfies the hypothesis of Lemma 4.3. Since convolutions of logarithmically concave functions are logarithmically concave $([11])$ and $K$ is the $n$-fold convolution of $\left(\operatorname{sech}^{2}(x)\right)^{\wedge}$; it follows that $K$ is logarithmically concave, and hence $K \in P F(2)$ by Lemma 4.3. Moreover, as seen above, $\hat{\varphi}(x)>0$ for all $x \in \mathbb{R}$. An application of Theorems 3.1 and 3.2 thus yields the desired result.

The next order of business is to determine the stability of the solitary waves of Theorem 4.6 by computing the sign of the quantities $I$. Unfortunately, the use of Lemma 4.1 requires formulas for differentiable families of solitary waves, which are not available in the present instance. Instead, $I$ will be computed by means of a spectral analysis of the operators $T_{0}$ and $S_{0}$ introduced above in Section 2. This analysis is carried out in the next three lemmas. The notation

$$
\lambda_{m}=\frac{\Gamma(r+m)}{\Gamma(r+1)} \cdot \frac{\Gamma(r+2 n+1)}{\Gamma(r+2 n+m)} \quad(m \geq 0)
$$

will be used throughout.

Lemma 4.7. For any integer $m \geq 0$, there exist constants $c_{m j} \quad(0 \leq j \leq m-1)$, depending only on $n$ and $p$, such that

$$
\partial^{m}\left(\frac{\varphi^{p+1}}{p+1}\right)=\varphi^{p}\left\{\left(\frac{1}{\lambda_{m}}\right)\left(\partial^{m} \varphi\right)+\sum_{j=0}^{m-1} c_{m j}\left(\partial^{j} \varphi\right)\right\}
$$

Proof. The proof is by induction. The statement of the Lemma clearly holds for $m=0$. Assume that it holds for $m$. A simple computation shows that for any integer $j \geq 0$, there exist constants $\beta_{j \ell} \quad(0 \leq \ell \leq j)$ such that

$$
(\partial \varphi)\left(\partial^{j} \varphi\right)=\varphi\left\{\left(\frac{r}{r+j}\right)\left(\partial^{j+1} \varphi\right)+\sum_{\ell=0}^{j} \beta_{j \ell}\left(\partial^{\ell} \varphi\right)\right\}
$$


Hence, by the inductive hypothesis,

$$
\begin{aligned}
\partial^{m+1}\left(\frac{\varphi^{p+1}}{p+1}\right)= & \partial \partial^{m}\left(\frac{\varphi^{p+1}}{p+1}\right) \\
= & \left(\frac{1}{\lambda_{m}}\right) \partial\left(\varphi^{p}\left(\partial^{m} \varphi\right)\right)+\sum_{j=0}^{m-1} c_{m j} \partial\left(\varphi^{p}\left(\partial^{j} \varphi\right)\right) \\
= & \left(\frac{1}{\lambda_{m}}\right)\left[p \varphi^{p-1}(\partial \varphi)\left(\partial^{m} \varphi\right)+\varphi^{p}\left(\partial^{m+1} \varphi\right)\right] \\
& +\sum_{j=0}^{m-1} c_{m j}\left[p \varphi^{p-1}(\partial \varphi)\left(\partial^{j} \varphi\right)+\varphi^{p}\left(\partial^{j+1} \varphi\right)\right] \\
= & \left(\frac{1}{\lambda_{m}}\right)\left(\frac{p r}{r+m}+1\right) \varphi^{p}\left(\partial^{m+1} \varphi\right) \\
& +\varphi^{p} \sum_{j=0}^{m-1}\left\{\left(\frac{p c_{m j} r}{r+j}+1\right)\left(\partial^{j+1} \varphi\right)+\sum_{\ell=0}^{j} p c_{m j} \beta_{j \ell}\left(\partial^{\ell} \varphi\right)\right\} .
\end{aligned}
$$

Since $\left(\frac{1}{\lambda_{m}}\right)\left(\frac{p r}{r+m}+1\right)=\left(\frac{1}{\lambda_{m+1}}\right)$, this proves the statement of the Lemma for $(m+1)$.

Lemma 4.8. For any integer $m \geq 0$, there exist constants $\gamma_{m i} \quad(0 \leq i \leq m)$, depending only on $n$ and $p$, such that the function $q_{m}=\sum_{i=0}^{m} \gamma_{m i}\left(\partial^{i} \varphi\right)$ satisfies $\left(M_{n, p}+C_{n, p}\right)\left(q_{m}\right)=\left(\frac{1}{\lambda m}\right) \varphi^{p} q_{m}$.

Proof. Define the matrix $G=\left\{g_{i j}\right\}_{0 \leq i, j \leq m}$ by

$$
g_{i j}=\left\{\begin{array}{lll}
c_{i j} & \text { if } & 0 \leq j \leq i-1 \\
\left(\frac{1}{\lambda_{i}}\right) & \text { if } \quad j=i \\
0 & \text { if } \quad i+1 \leq j \leq m
\end{array}\right.
$$

By Lemma 4.7, if $0 \leq i \leq m$ then

$$
\partial^{i}\left(\frac{\varphi^{p+1}}{p+1}\right)=\varphi^{p} \sum_{j=0}^{m} g_{i j}\left(\partial^{j} \varphi\right) .
$$

Since $G$ is a lower-diagonal matrix with diagonal entries $g_{i i}=\frac{1}{\lambda_{i}}$, then $\frac{1}{\lambda_{i}}$ is an eigenvalue of $G$ for each $0 \leq i \leq m$. Define $\left(\gamma_{m 0}, \ldots, \gamma_{m m}\right)$ to be a left eigenvector of $G$ for the eigenvalue $\frac{1}{\lambda_{m}}$, so that $\sum_{i=0}^{m} \gamma_{m i} g_{i j}=\left(\frac{1}{\lambda_{m}}\right) \gamma_{m j}$ for each $0 \leq j \leq m$. 
Then by (4.2),

$$
\begin{aligned}
\left(M_{n, p}+C_{n, p}\right)\left(q_{m}\right) & =\sum_{i=0}^{m} \gamma_{m i} \partial^{i}\left(M_{n, p}+C_{n, p}\right)(\varphi) \\
& =\sum_{i=0}^{m} \gamma_{m i} \partial^{i}\left(\frac{\varphi^{p+1}}{p+1}\right)=\left(\varphi^{p}\right) \sum_{i=0}^{m} \gamma_{m i} \sum_{j=0}^{m} g_{i j}\left(\partial^{j} \varphi\right) \\
& =\left(\varphi^{p}\right) \sum_{j=0}^{m}\left(\sum_{i=0}^{m} \gamma_{m i} g_{i j}\right)\left(\partial^{j} \varphi\right) \\
& =\left(\varphi^{p}\right) \sum_{j=0}^{m}\left(\frac{1}{\lambda_{m}} \gamma_{m j}\right)\left(\partial^{j} \varphi\right)=\frac{1}{\lambda_{m}} \varphi^{p} q_{m} .
\end{aligned}
$$

Now, as in Section 2, let $T_{0}: Y \rightarrow Y$ be the operator defined by $T_{0}(g)=$ $\left(M_{n, p}+C_{n, p}\right)^{-1}\left(\varphi^{p} g\right)$ for $g \in Y$.

Lemma 4.9. Let $\rho=r+n-\frac{1}{2}$. For each integer $m \geq 0$, the function $\zeta_{m}(x)=$ $\varphi(x) C_{m}^{\rho}(\tanh x)$ is an eigenfunction of $T_{0}$ for the eigenvalue $\lambda_{m}$. Furthermore, $\left\{\zeta_{m}\right\}_{m \geq 0}$ forms a complete set of eigenfunctions for $T_{0}$ in $Y$.

Proof. By Lemma 4.8, the $\left\{q_{m}\right\}$ are eigenfunctions (for distinct eigenvalues) of the self-adjoint operator $T_{0}$ in $Y$; hence the $\left\{q_{m}\right\}$ must be mutually orthogonal in $Y$. Write $q_{m}$ as $q_{m}(x)=\varphi(x) P_{m}(z)$, where $z=\tanh x$ and $P_{m}(z)$ is a polynomial in $z$ of degree $m$. Then for $m \neq \ell$, one has $0=\left\langle q_{m}, q_{\ell}\right\rangle_{Y}=\int_{-\infty}^{\infty} q_{m}(x) q_{\ell}(x) \varphi^{p}(x) d x=$ $\int_{-\infty}^{\infty} P_{m}(z) P_{\ell}(z)\left(1-z^{2}\right)^{r+n-1} d z=\left\langle P_{m}, P_{\ell}\right\rangle_{2, \rho}$. Hence from the uniqueness property of the Gegenbauer polynomials described in Section 2 , it follows that for each $m \geq 0$, $P_{m}$ is a constant multiple of $C_{m}^{\rho}$; and therefore that $q_{m}$ is a constant multiple of $\zeta_{m}$. This proves that $\zeta_{m}$ is an eigenfunction of $T_{0}$ for the eigenvalue $\lambda_{m}$.

It remains to prove that $\left\{\zeta_{m}\right\}_{m \geq 0}$ is complete in $Y$. Suppose $g \in Y$ is such that $\left\langle g, \zeta_{m}\right\rangle_{Y}=0$ for all $m \geq 0$. Define $h(z)$ for $-1<z<1$ by $h(z)=h(\tanh x) g(x)=$ $g(x)\left(1-\tanh ^{2} x\right)^{-r / 2}$. It is readily verified that $\|h\|_{2, \rho}=\|g\|_{Y}<\infty$, so $h \in L_{2, \rho}$; and that $\left\langle h, C_{m}^{\rho}\right\rangle_{2, \rho}=\left\langle g, \zeta_{m}\right\rangle_{Y}=0$ for all $m \geq 0$. Since $\left\{C_{m}^{\rho}\right\}_{m \geq 0}$ is a basis for $L_{2, \rho}$, it follows that $h=0$ in $L_{2, \rho}$, whence $g=0$ in $Y$.

Remark. Lemma 4.9 is related to the following amusing fact, which is a generalization of an elementary result from the theory of special functions: for every $\rho>-\frac{1}{2}$ and every integer $n \geq 1$, there is a linear differential operator $L$ of order $2 n$, with polynomial coefficients, whose eigenfunctions are the Gegenbauer polynomials $\left\{C_{m}^{\rho}\right\}_{m \geq 0}$. (In the notation of Lemma $4.9, L$ is the operator defined on functions $v$ of the variable $z=\tanh x$ by $(L v)(z)=(\varphi(x))^{-(p+1)}\left[M_{n, p}+C_{n, p}\right](\varphi(x) v(\tanh x))$.)

Theorem 4.10. The quantity I appearing in Theorem 4.6 is given by the formula

$$
a \sum_{j=0}^{\infty}\left(\frac{\lambda_{2 j}}{1-\lambda_{2 j}}\right)\left\{\frac{\Gamma(2 j+1) \cdot\left(2 j+n+r-\frac{1}{2}\right)}{\Gamma(2 j+2 n+2 r-1)}\right\}\left\{\frac{\Gamma(j+n) \Gamma\left(j+n+r-\frac{1}{2}\right)}{\Gamma(j+1) \Gamma\left(j+r+\frac{1}{2}\right)}\right\}^{2},
$$


where $a=\left(\frac{2^{n+r-1} \Gamma(r)}{\pi \Gamma(n)}\right)^{2}$.

Proof. Define $e_{i}=\sqrt{\lambda_{i}}\left(\frac{\widehat{\zeta}_{i}}{\left\|\zeta_{i}\right\|_{Y}}\right)$ for $i \geq 0$. Then by Lemma 4.9 and Proposition 2.6, $\left\{e_{i}\right\}_{i \geq 0}$ is a complete orthonormal set of eigenfunctions for $S_{0}$ in $X$. Define a function $\eta$ by

$$
\eta=\sum_{i=0}^{\infty}\left(\frac{1}{1-\lambda_{i}}\right)\left\langle\frac{\hat{\varphi}}{w_{0}}, e_{i}\right\rangle_{X, 0} e_{i} .
$$

(Here and in the remainder of this proof, the notational convention is adopted that $\frac{0}{0}=0$.) Since

$$
\begin{gathered}
\sum_{i=0}^{\infty}\left(\frac{1}{1-\lambda_{i}}\right)^{2}\left|\left\langle\frac{\widehat{\varphi}}{w_{0}}, e_{i}\right\rangle_{X, 0}\right|^{2} \leq A \sum_{i=0}^{\infty}\left|\left\langle\frac{\widehat{\varphi}}{w_{0}}, e_{i}\right\rangle_{X, 0}\right|^{2} \\
=A\left\|\frac{\hat{\varphi}}{w_{0}}\right\|_{X, 0}^{2}=A \int_{-\infty}^{\infty}|\hat{\varphi}|^{2} d x=A\|\varphi\|_{0}^{2}
\end{gathered}
$$

(where $A=\sup _{i \neq 1}\left|\frac{1}{1-\lambda_{i}}\right|^{2}$ ); the series for $\eta$ converges in $X$, and so $\eta \in X \subseteq L^{2}$.

Now choose $\psi \in L^{2}$ so that $\hat{\psi}=\eta$. Then

$$
\begin{aligned}
(\mathcal{L} \psi)^{\wedge} & =\left[\left(M_{n, p}+C_{n, p}\right) \psi-\varphi^{p} \psi\right]^{\wedge}=w_{0}\left(\eta-T_{0} \eta\right) \\
& =w_{0} \sum_{i=0}^{\infty}\left(\frac{1}{1-\lambda_{i}}\right)\left\langle\frac{\hat{\varphi}}{w_{0}}, e_{i}\right\rangle_{X, 0}\left(e_{i}-T_{0} e_{i}\right) \\
& =w_{0} \sum_{i=0}^{\infty}\left\langle\frac{\hat{\varphi}}{w_{0}}, e_{i}\right\rangle_{X, 0} e_{i}=\hat{\varphi} .
\end{aligned}
$$

Hence $\mathcal{L} \psi=\varphi$, and so $I=\langle\psi, \varphi\rangle_{0}$.

Applying the inverse Fourier transform to $\eta$ gives

$$
\psi=\sum_{i=0}^{\infty}\left(\frac{\lambda_{i}}{1-\lambda_{i}}\right)\left(\int_{-\infty}^{\infty} \varphi(t) \zeta_{i}(t) d t\right) \frac{\zeta_{i}}{\left\|\zeta_{i}\right\|_{Y}^{2}} .
$$

Therefore

$$
\begin{aligned}
I=\langle\psi, \varphi\rangle_{0} & =\sum_{i=0}^{\infty}\left(\frac{\lambda_{i}}{1-\lambda_{i}}\right) \frac{\left(\int_{-\infty}^{\infty} \varphi(t) \zeta_{i}(t) d t\right)^{2}}{\left\|\zeta_{i}\right\|_{Y}^{2}} \\
& =\sum_{i=0}^{\infty}\left(\frac{\lambda_{i}}{1-\lambda_{i}}\right) \frac{\left(\int_{-\infty}^{\infty} C_{i}^{\rho}(\tanh x) \operatorname{sech}^{2 r}(x) d x\right)^{2}}{\left.\int_{-\infty}^{\infty}\left(C_{i}^{\rho}(\tanh x)\right)^{2} \operatorname{sech}^{2 n+2 r}(x) d x\right)} \\
& =\sum_{i=0}^{\infty}\left(\frac{\lambda_{i}}{1-\lambda_{i}}\right) \frac{\left(\int_{-1}^{1} C_{i}^{\rho}(z)\left(1-z^{2}\right)^{r-1} d z\right)^{2}}{\left(\int_{-1}^{1}\left(C_{i}^{\rho}(z)\right)^{2}\left(1-z^{2}\right)^{n+r-1} d z\right)} .
\end{aligned}
$$

The statement of the Theorem now follows from formulas (2.5) and (2.6). 
No simple relation has yet been found between the parameters $n$ and $p$ which is equivalent to the condition that the series for $I$ in the preceding theorem sum to a negative value. However, for a given choice of $n$ and $p$, it is not hard to numerically determine the sign of $I$. Let the $j^{t h}$ term in the series be denoted by $b_{j}$. Since $b_{0}<0$ and $b_{j}>0$ for $j \geq 1$, it suffices to decide whether $\sum_{j=1}^{\infty} b_{j}<\left|b_{0}\right|$. This task is simplified by the rapid decay of $b_{j}$ (by Stirling's formula, $b_{j} \sim j^{-2 r-1}$ as $j \rightarrow \infty$ ). As an example, consider the solitary-wave solution $u(x, t)=\operatorname{sech}^{4}\left(x-\frac{12}{35} t\right)$ of the equation

$$
u_{t}+u u_{x}+\frac{13}{420} u_{x x x}-\frac{1}{1680} u_{x x x x x}=0 .
$$

Here $n=2$ and $p=1$, so

$$
b_{j}=\frac{1680\left(2 j+\frac{11}{2}\right)(j+1)^{2}\left(j+\frac{9}{2}\right)^{2}(2 j) !}{\{(2 j+4)(2 j+5)(2 j+6)(2 j+7)-1680\}(2 j+10) !}
$$

and one finds that $\left|b_{0}\right|=2\left\{\frac{(11 / 2)}{10 !}\right\}\left\{\frac{81}{4}\right\} \approx 6.14 \times 10^{-5}$, while $\sum_{j=1}^{\infty} b_{j} \approx 5.05 \times 10^{-6}$. Hence this solitary wave is stable.

A simple Fortran program was written to find the sign of $I$ for various values of $n$ and $p$. As mentioned earlier, it was already known that, if $n=1$, then $I<0$ if and only if $p<4$. For $n=2$, the value of $p$ at which $I$ changes sign was found to be approximately 4.82, while for $n=3$ it is approximately 5.26 .

An interesting example of an unstable solitary wave occurs in the case $n=2$ and $p=5$. As shown in the proof of Theorem 4.6, the operator $\mathcal{L}$ associated with the solitary wave $\varphi$ satisfies properties (P1)-(P3) of Theorem 3.1; whereas the remarks of the preceding paragraph show that $I>0$ in this case. It therefore follows from the results of [24] that $\varphi$ is unstable. On the other hand, since $p<4 n$, Theorem 2.1(b) shows that the equation

$$
u_{t}+u^{5} u_{x}-\left(M_{2,5} u\right)_{x}=0
$$

has global solutions in $H^{s}$ for any $s>\frac{3}{2}$. Thus, although $\varphi$ is unstable, solutions with initial data near to $\varphi$ do not blow up in finite time. (By contrast, when $n=1$, unstable solitary waves occur only when $p \geq 4$, and in these cases numerical simulations suggest that nearby solutions to $\varphi$ do blow up [9].) While unstable solitary waves for other globally well-posed equations were found in [24], this appears to be the first such example known for an equation of type (2.1).

\section{Solitary Waves with Positive Fourier Transforms.}

In this section, some results are proved which suggest that the hypothesis of Theorem 3.2 (and hence also the hypotheses (P1)-(P3) of Theorem 3.1) holds for solitary-wave solutions of broad classes of equations of type (2.1). These results will be stated in the context of the existence theory for solitary waves introduced by $\mathrm{M}$. Weinstein in [26]. Let $C>-b$ be given, and define a functional $J_{C}(v)$ for $v \in H^{\mu / 2}$ by

$$
J_{C}(v)=\frac{\left(\int_{-\infty}^{\infty} v(x)[(M+C) v(x)] d x\right)}{\left(\int_{-\infty}^{\infty} v^{p+2}(x) d x\right)^{2 /(p+2)}} .
$$


If $J_{C}(v)$ has a critical point at $v=v_{0}$, then a computation of the gradient of $J_{C}$ at $v_{0}$ shows that $v_{0}$ is, up to a constant multiple, a solution of the solitary wave equation (2.3).

In what follows, we will make the following assumption:

(E) There exists $v_{0} \in H^{\mu / 2}$ such that $J_{C}\left(v_{0}\right)=\inf \left\{J_{C}(v): v \in H^{\mu / 2}\right\}$.

Under certain assumptions on the symbol $\alpha(k)$ of $M$, the validity of (E) may be established by means of the method of "concentrated compactness". For details the reader is referred to [26].

Theorem 5.1. Suppose (E) holds. Then there exists a solution $\varphi$ of (2.3) such that $\widehat{\varphi}(k) \geq 0$ for all $k \in \mathbb{R}$. Moreover, if $\alpha(k)$ is a non-decreasing function of $|k|$, then $\varphi$ may be chosen so that $\widehat{\varphi}(k)$ is a positive, non-increasing function of $|k|$.

The proof of Theorem 5.1 requires the following theorem of F. Riesz concerning convolutions of "rearrangements" of functions. Recall that if $f$ is a measurable function on $\mathbb{R}$ such that meas $\{x: f(x) \geq y\}<\infty$ for all $y>0$, then there exists a positive, even, measurable function $f^{*}$ on $\mathbb{R}$ which is a nonincreasing function of $|x|$, and which satisfies meas $\left\{x: f^{*}(x) \geq y\right\}=\operatorname{meas}\{x:|f(x)| \geq y\}$ for all $y>0$, (see, e.g. [20]).

Theorem 5.2 (F. Riesz). Let $f_{1}, \ldots, f_{n}$ be measurable functions on $\mathbb{R}$ such that meas $\left\{x: f_{i}(x) \geq y\right\}<\infty$ for all $y>0$ and all $1 \leq i \leq n$. Then

$$
\left|\left(f_{1} * f_{2} * \cdots * f_{n}\right)(0)\right| \leq\left[\left(f_{1}^{*}\right) *\left(f_{2}^{*}\right) * \cdots *\left(f_{n}^{*}\right)\right](0)
$$

in the sense that if the right-hand side is finite, then the left-hand side exists and the inequality holds.

A proof of Theorem 5.2 for the case $n=3$ is given in [22]; along with the sketch of the (inductive) proof for $n \geq 3$. A complete proof of a more general result may be found in [12].

The next result is elementary.

Lemma 5.3. Suppose $f(x)$ is an even function and is non-decreasing as a function of $|x|$. If $E \subseteq \mathbb{R}$ has measure $2 q$, then $\int_{E} f(x) d x \geq \int_{F} f(x) d x$, where $F=[-q, q]$.

Proof. Since $\int_{E \backslash F} h(x) d x \geq h(q) \operatorname{meas}(E \backslash F)=h(q) \operatorname{meas}(F \backslash E) \geq$ $\int_{F \backslash E} h(x) d x$, it follows that $\int_{E} h(x) d x=\int_{E \cap F} h(x) d x+\int_{E \backslash F} h(x) d x \geq$ $\int_{E \cap F} h(x) d x+\int_{F \backslash E} h(x) d x=\int_{F} h(x) d x$.

Proof of Theorem 5.1. Let $\left(H^{\mu / 2}\right)^{\wedge}$ denote the space $\left\{\hat{v}: v \in H^{\mu / 2}\right\}=$ $\left\{\hat{v}: \int_{-\infty}^{\infty}|\hat{v}(k)|^{2}\left(1+|k|^{2}\right)^{\mu} d k<\infty\right\}$. By the Sobolev Embedding Theorem, if $v \in H^{\mu / 2}$ then $v \in L^{p+2}$, and hence $\int_{-\infty}^{\infty} v^{p+2} d x=\left(v^{p+2}\right)^{\wedge}(0)=(\hat{v} * \cdots * \hat{v})(0)$ (where the convolution is performed $p+1$ times). Therefore if the functional $\widetilde{J}_{C}$ is defined on $\left(H^{\mu / 2}\right)^{\wedge}$ by

$$
\widetilde{J}_{C}(\hat{v})=\frac{\int_{-\infty}^{\infty}|\hat{v}(k)|^{2}(C+\alpha(k)) d k}{(\hat{v} * \cdots * \hat{v}(0))^{2 / p+2}},
$$


then $\widetilde{J}_{C}(\hat{v})=J_{C}(v)$ for all $v \in H^{\mu / 2}$. Let $v_{0}$ be a minimizer of $J_{C}(v)$ in $H^{\mu / 2}$, so that $\hat{v}_{0}$ is a minimizer of $\widetilde{J}_{C}(\hat{v})$ in $H^{\mu / 2}$. Choose $v_{1} \in L^{2}$ such that $\hat{v}_{1}=\left|\hat{v}_{0}\right|$. Clearly $v_{1} \in H^{\mu / 2}$ and $\hat{v}_{1} * \cdots * \hat{v}_{1}(0) \geq\left|\hat{v}_{0} * \cdots * \hat{v}_{0}(0)\right|$, so $J_{C}\left(v_{1}\right)=\widetilde{J}_{C}\left(\hat{v}_{1}\right) \leq$ $\widetilde{J}_{C}\left(\hat{v}_{0}\right)=J_{C}\left(v_{0}\right)$. Hence $v_{1}$ is also a minimizer of $J_{C}(v)$, and it follows that there exists a constant $a \in \mathbb{R}$ such that $\varphi=a v_{1}$ is a solution of (2.3). Since $\hat{\varphi}=a \hat{v}_{1}$ is nonnegative on $\mathbb{R}$, this proves the first assertion of the Theorem.

Now assume that $\alpha(k)$ is a non-decreasing function of $|k|$, and as above let $v_{0}$ be a minimizer of $J_{C}(v)$ in $H^{\mu / 2}$. Choose $v_{2} \in L^{2}$ such that $\hat{v}_{2}=\hat{v}_{0}^{*}$. It is claimed that $\widetilde{J}_{C}\left(\hat{v}_{2}\right) \leq \widetilde{J}_{C}\left(\hat{v}_{0}\right)$. To see this, notice that Theorem 5.2 implies $\hat{v}_{2} * \cdots * \hat{v}_{2}(0) \geq$ $\left|\hat{v}_{0} * \cdots * \hat{v}_{0}(0)\right|$. Hence to prove the claim it suffices to show that $\int_{-\infty}^{\infty}\left|\hat{v}_{2}(k)\right|^{2}(C+$ $\alpha(k)) d k$

$\int_{-\infty}^{\infty}\left|\hat{v}_{0}(k)\right|^{2}(C+\alpha(k)) d k$. Define $\beta(y)=\int_{\left\{k: \hat{v}_{0}^{*}(k) \geq y\right\}}(C+\alpha(k)) d k$ and $\gamma(y)=\int_{\left\{k:\left|\hat{v}_{0}(k)\right| \geq y\right\}}(C+\alpha(k)) d k$ for $y>0$. From Lemma 5.3 it follows that $\gamma(y) \geq \beta(y)$ for all $y>0$. Using standard formulas connecting Lebesgue integrals and Riemann-Stieltjes integrals [27], one then obtains $\int_{-\infty}^{\infty}\left|\hat{v}_{0}^{*}(k)\right|^{2}(C+$ $\alpha(k)) d k=-\int_{0}^{\infty} y^{2} d \beta(y)=\int_{0}^{\infty} \beta(y) 2 y d y \leq \int_{0}^{\infty} \gamma(y) 2 y d y=-\int_{0}^{\infty} y^{2} d \gamma(y)=$ $\int_{-\infty}^{\infty}\left|\hat{v}_{0}(k)\right|^{2}(C+\alpha(k)) d k$; and so the claim is proved. It follows from the claim, as in the preceding paragraph, that there exists $a \in \mathbb{R}$ for which $\varphi=a v_{2}$ is a solution of $(2.3)$.

Since $\hat{\varphi}=a \hat{v}_{2}=a \hat{v}_{0}^{*}$ is a nonincreasing function of $|k|$, it remains only to show that $\hat{\varphi}$ is everywhere positive. Suppose that this is not the case. Then the support of $\hat{\varphi}$ is a finite interval $[-d, d]$. On the other hand, the support of the $(p+1)$-fold convolution $\hat{\varphi} * \cdots * \hat{\varphi}$ strictly contains $[-d, d]$, so that $(C+\alpha(k)) \hat{\varphi}$ cannot equal $\frac{1}{p+1}(\hat{\varphi} * \cdots * \hat{\varphi})$. This then contradicts $(2.3)$.

To prove that the solitary wave $\varphi$ of Theorem 5.1 satisfies hypotheses $(\mathrm{P} 1)-(\mathrm{P} 3)$ of Theorem 3.1, it would suffice, by Theorem 3.2, to show that $\left(\varphi^{p}\right)^{\wedge}$ is in the class $P F(2)$. Unfortunately, Theorem 5.1 falls short of this assertion. The following partial result may however be obtained. (Similar results, requiring the assumption that $\varphi$ be positive, appear in [3] and [26].)

Corollary 5.5. Suppose $\alpha(k)$ is a non-decreasing function of $|k|$. Then for the solitary wave $\varphi$ defined in Theorem 5.1, the operator $\mathcal{L}$ given by (2.4) satisfies properties (P1) and (P2) of Theorem 3.1.

Proof. Since $\hat{\varphi}$ is strictly positive, so also is the function $K=\left(\varphi^{p}\right)^{\wedge}=\hat{\varphi} * \cdots * \hat{\varphi}$. Consequently, by a standard argument in spectral theory (see e.g. the proof of Lemma 8 of [2]), the greatest eigenvalue $\lambda_{0}(\theta)$ of the operator $S_{\theta}$ must be simple and positive, for any $\theta \geq 0$. Moreover, any corresponding eigenfunction $\psi_{0}=\psi_{0}(\theta)$ must be of one sign on $\mathbb{R}$. Now, differentiating (2.3) and applying the Fourier transform to both sides leads to the result

$$
(C+\alpha(k)) \cdot\left(\frac{d \varphi}{d x}\right)^{\wedge}=\left(\varphi^{p}\right)^{\wedge} *\left(\frac{d \varphi}{d x}\right)^{\wedge} ;
$$

from which it follows that $\left(\frac{d \varphi}{d x}\right)^{\wedge}$ is an eigenfunction of $S_{0}$ for the eigenvalue $\lambda=1$. Since $\left(\frac{d \varphi}{d x}\right)^{\wedge}(k)=i k \hat{\varphi}(k)$ is not of one sign on $\mathbb{R}$, one must have $\lambda_{0}(0) \neq 1$, so 
$\lambda_{0}(0)>1$. From (3.1) (still valid in this situation for $i=0$ ) and (3.2) it then follows that there exists a unique $\theta_{0} \in(0, \infty)$ such that $\lambda_{0}\left(\theta_{0}\right)=1$. Property (P1) then follows from Corollary (2.3) as in the proof of Theorem 3.2.

Once (P1) has been proved, (P2) follows from the fact that $\varphi$ is a minimizer of the functional $J_{C}$; as was demonstrated by Weinstein in Proposition 4.2 of [26].

\section{Acknowledgements.}

Research for this article was done while the author was in residence at the Institute for Mathematics and its Applications at the University of Minnesota. He is indebted to Dan Dix and John Hunter for stimulating conversations, and to Willard Miller for help with the special functions used in section 4 .

\section{REFERENCES}

[1] L. Abdelouhab, J.L. Bona, M. Felland and J.-C. Saut, Non-local models for nonlinear, dispersive waves, Physica D, to appear.

[2] J.P. Albert and J.L. Bona, Total positivity and the stability of internal waves in stratified fluids of finite depth, IMA Journal of Appl. Math., to appear.

[3] J.P. Albert, J.L. Bona, and D.B. Henry, Sufficient conditions for stability of solitary-wave solutions of model equations for long waves, Physica 24D (1987), 343.

[4] T.B. Benjamin, Internal waves of permanent form in fluids of great depth, J. Fluid Mech. 29 (1967), 559.

[5] _ The stability of solitary waves, Proc. Royal Soc. London A 328 (1972), 153.

[6] T.B. Benjamin, J.L. Bona and D.K. Bose, Solitary-wave solutions of nonlinear problems, Phil. Trans. Royal Soc. London 331 (1990), 195.

[7] D.P. Bennett, J.L. Bona, R.W. Brown, S.E. Stansfield and J.D. Stroughair, The stability of internal solitary waves, Math. Proc. Camb. Phil. Soc. 94 (1983), 351.

[8] J.L. Bona, On the stability theory of solitary waves, Proc. Royal Soc. London A 344 (1975), 363.

[9] J.L. Bona, V.A. Dougalis and O.A. Karakashian, Fully discrete Galerkin methods for the Korteweg-de Vries equation, Comp. Math. Appls. 12A (1986), 859.

[10] J.L. Bona, P.E. Souganides and W.A. Strauss, Stability and instability of solitary waves of Korteweg-de Vries type, Proc. Royal Soc. London A 411 (1987), 395.

[11] H.J. Brascamp and E.H. Lieb, On extensions of the Brunn-Minkowski and Prékopa-Leindler theorems, including inequalities for log concave functions, and with an application to the diffusion equation, J. Funct. Anal. 22 (1976), 366.

[12] H.J. Brascamp, E.H. Lieb and J.M. Luttinger, A general rearrangement inequality for multiple integrals, J. Funct. Anal. 17 (1974), 227.

[13] M. Grillakis, J. Shatah and W.A. Strauss, Stability theory of solitary waves in the presence of symmetry I, J. Funct. Analysis 74 (1987), 160.

[14] Stability theory of solitary waves in the presence of symmetry II, preprint.

[15] L.K. Hua, Harmonic Analysis of Functions of Several Complex Variables in the Classical Domains, A.M.S. Translations of Math. Monographs 6 (1963), Amer. Math. Soc., Providence, RI.

[16] R.I. Joseph, Solitary waves in a finite depth fluid, J. Phys. A 10 (1977), L225.

[17] S. Karlin, Total Positivity, Stanford Univ. Press, Stanford, CA, 1968.

[18] T. Kato, Perturbation Theory for Linear Operators, 2nd ed., Springer, Berlin, 1976.

[19] _ On the Cauchy problem for the (generalized) Korteweg-de Vries equation, Studies in Appl. Math., Advances in Math. Supplementary Studies Vol. 8, Academic Press, NY (1983), 93.

[20] B. Kawohl, Springer Lecture Notes no. 1150, Springer, Berlin, 1985. 
[21] W. Magnus and E. Oberhettinger, Formulas and Theorems for the Special Functions of Mathematical Physics, Springer, NY, 1966.

[22] F.W.J. Olver, Asymptotics and Special Functions, Academic Press, NY, 1974.

[23] F. Riesz, Sur une inégalité intégrale, J. London Math. Soc. 5 (1930), 162.

[24] P.E. Souganides and W.A. Strauss, Instability of a class of dispersive solitary waves, preprint.

[25] M. Weinstein, Lyapunov stability of ground states of nonlinear dispersive evolution equations, Commun. Pure Appl. Math. 39 (1986), 51.

[26] _ Existence and dynamic stability of solitary-wave solutions of equations arising in long wave propagation, Commun. Partial Diff. Eqns. 12 (1987), 1133.

[27] R.L. Wheeden and A. Zygmund, Measure and Integral: An Introduction to Real Analysis, Marcel Dekker, NY, 1977. 